\title{
Research on image and video transmission mechanism of the Android platform based on $4 \mathrm{G}$
}

\author{
Liu Juanjuan', Liu Zhengguang ${ }^{2}$, Zhao Zhigang ${ }^{1}$ \\ ${ }^{1}$ College of information and automation, Tianshi College,Tianjin,300232 China \\ ${ }^{2}$ Electrical Engineering \&Automation, Tianjin University, Tianjin, 300072 China
}

\section{Keywords: Video data, Data Transmission, Network Protocol, New Era of Networks}

\begin{abstract}
Dynamic mapping method based on unequal importance of the video data is put forward in this paper. First of all, in terms of the three types of data A, B and C in data rate in H.264 using data partitioning, calculate their transmission error or loss within one Group of Picture (GOP) and the average distortion of current frame and following frames caused by them. Then, separate importance factors of the data depending on the degree of distortion they have made and determine the transmission priority of the data. Finally, map various video data to different EDCA queues according to the transmission priority and situation of the queues and obtain the best video receiving quality the time the channel utilization is enhanced.
\end{abstract}

\section{Introduction}

The new Wireless Local Area Network (WLAN) is of two development routes. The first is about two new physical layer protocols IEEE 802.11g and 802.11n with the former providing a rate at $54 \mathrm{Mbps}$ and the latter at 100Mbps. The second is to MAC Layer Protocol IEEE 802.11e ensuring of Quality of Service (QoS) guarantee, which provides differentiated service for audio, video and general data and improve the ability of 802.11 in offering QoS for multimedia business. The new MAC-layer transport mechanism, i.e. the Enhanced Distributed Channel Access (EDCA) is proposed in 802.11e in which four queues are defined: $A C_{0} \sim A C_{3}$. EDCA dictates the parameters of different media access priorities for the four queues, making $A C_{3}$ be of the highest priority, $A C_{2}$ the second, $A C_{1}$ the third and $A C_{0}$ the last. By default, data packet of voice traffic is mapped to $A C_{3}$, visual data packet to $A C_{2}$, and best-effort data packet to $A C_{1}$, with other data packets being the background data mapped to $A C_{0}$.In this case, the audio/visual data packet will be transported in during transit firstly with a short waiting time and then provide QoS for media business.

\section{Model for the importance of $\mathbf{H . 2 6 4}$ video data}

Assume the length of GOP is $L$ and the encoding structure is IPP...P, i.e. I-frame and $L-1$ P-frame. It is common that the first frame in GOP must be IDR frame (the independently coded frame) and its loss can result in that the following $\mathrm{P}$ frames fail to decode correctly until the next synchronous identifier is found. Provided that the IDR frame cannot get lost, analyze only the distortion triggered by the partitioning loss of DPA, DPB and DPC in the following L-1 P-frame.

Assume the number of pixels in each frame is $N_{f}$, with $f_{i}^{m}$ representing the original pixel value of $\mathrm{m}^{\text {th }}$ pixel of the $\mathrm{i}^{\text {th }}$ frame. $\hat{f}_{i}^{m}$ refers to restored pixel value of $f_{i}^{m}$ at the encoder side and refers to restored pixel value of $f_{i}^{m}$ at the decoder side. Express source coding with distortion, channel distortion and total distortion of the $i^{\text {th }}$ frame with Ds(i), Dc(i) and D(i) and apply Mean Square Error (MSE) as the distortion criteria, then the calculation formula for distortion is:

$$
\begin{aligned}
& D_{s}(i)=\frac{1}{N_{f}} \sum_{m=1}^{N_{f}}\left(f_{i}^{m}-\hat{f}_{i}^{m}\right)^{2} \\
& D_{c}(i)=\frac{1}{N_{f}} \sum_{m=1}^{N_{f}}\left(\hat{f}_{i}^{m}-\tilde{f}_{i}^{m}\right)^{2} \\
& D(i)=\frac{1}{N_{f}} \sum_{m=1}^{N_{n}}\left(f_{i}^{m}-\tilde{f}_{i}^{m}\right)^{2}
\end{aligned}
$$

The relation among total distortion, source coding with distortion and channel distortion is: 
$D(i)=D_{s}(i)+D_{c}(i)+\frac{2}{N_{f}} \sum_{m=1}^{N_{f}}\left(f_{i}^{m}-\hat{f}_{i}^{m}\right)\left(\hat{f}_{i}^{m}-\tilde{f}_{i}^{m}\right)$

Before analyzing the influence from data loss of the DPA, DPB and DPC in $i^{\text {th }}$ frame on $i^{\text {th }}$ and the following frames, the error concealment method below is taken to make the decoding proceed smoothly: 1). If the DPA of $\mathrm{i}^{\text {th }}$ frame get lost, adopt the MacroBlock (MB) data of the same position with $\mathrm{i}-1^{\text {th }}$ frame to conceal. 2). If the DPB of $\mathrm{i}^{\text {th }}$ frame get lost, as for intraframe coding, adopt the MB data of the same position with $\mathrm{i}-1^{\text {th }}$ frame to conceal. 3). If the DPC of $i^{\text {th }}$ frame get lost, apply the Motion Vector (MV) information parsed by DPA to obtain the prediction data from reference frame to conceal.

Assume $D(i, j, k)$ refers to the $\mathrm{j}^{\text {th }}$ distortion caused by the $\mathrm{k}^{\text {th }}$ partitioning loss of the $\mathrm{i}^{\text {th }}$ frame. Wherein $1 \leq \mathrm{i} \leq \mathrm{L}-1,0 \leq \mathrm{j} \leq \mathrm{L}-1, \mathrm{k}=\{0,1,2\}$ and three values of $\mathrm{k}$ refer to DPA, DPB and DPC respectively. In order to acquire degree of influence from the loss of data partioning parts of ith frame on restoration quality of GOP, calculate the distortion variation value of each frame on two occasions when the data is received correctly and lost, and then take the average distortion variation value $\overline{\Delta D}(i, k)$ as the degree of influence on reconstruction quality of GOP from the current data partitioning. The formula for average distortion variation value $\overline{\Delta D}(i, k)$ of GOP caused by the DPA of $i^{\text {th }}$ frame (i.e. the $0^{\text {th }}$ partitioning of $i^{\text {th }}$ frame), the DPB (i.e. the $1^{\text {th }}$ partitioning of $i^{\text {th }}$ frame) and the DPC (i.e. the $2^{\text {th }}$ partitioning of $i^{\text {th }}$ frame) on two occasions when the data is received correctly and lost is as below:

$$
\begin{aligned}
& \overline{\Delta D}(i, k)=\frac{1}{L} \sum_{j=0}^{L-1}\left[D(i, j, k)-D_{s}(j)\right], k=\{0,1,2\} \\
& \overline{\Delta D}(i, k)=\frac{1}{L N_{f}} \sum_{m=i}^{L-1} \sum_{p=1}^{N_{k}} 2\left(f_{m}^{p}-\hat{f}_{m}^{p}\right)\left(\hat{f}_{m}^{p}-\tilde{f}_{m}^{p}\right)+\left(\hat{f}_{m}^{p}-\tilde{f}_{m}^{p}\right)^{2}
\end{aligned}
$$

$\overline{\Delta D}(i, k, 0)$ and $\overline{\Delta D}(i, k, 1)$ refer to the variations of distortion introduced by all the intraframe MBs and the interframe MBs of ith frame respectively under two circumstances when the data partioning part $\mathrm{DP} k$ is the correctly received and lost, then the formula (6) is:

$$
\overline{\Delta D}(i, k)=\frac{1}{L N_{f}} \sum_{m=i}^{L-1}[\overline{\Delta D}(i, k, 0)+\overline{\Delta D}(i, k, 1)]
$$

In view of features of the three partitioned data DPA, DPB and DPC, if the DPA of $i^{\text {th }}$ frame is lost, all the MB data of $i^{\text {th }}$ frame will get lost and data in the whole frame will need to be consealed with the above-mentioned error concealment method. On account that DPB contains the information on difference value of the intra-coded $M B$, intra-frame $M B$ of the $i^{\text {th }}$ frame needs to be concealed with the above-mentioned error concealment method if DPB gets lost. Inter-frame MB of the $\mathrm{i}^{\text {th }}$ frame also requires consealment with the above-mentioned error concealment method once DPC gets lost. For frames following the $i^{\text {th }}$ frame within GOP, the distortion of $i^{\text {th }}$ frame consealed by errors can diffuse to the subsequent frames because of the dependency among frames. After the data partitioning parts of $i^{\text {th }}$ frame get lost individually, the distortion variation values for $i+1^{\text {th }}$ frame and the subsequent frames can be calculated with the formula below through recursive algorithm:

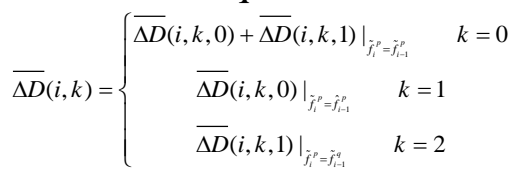

$\overline{\Delta D}(i+1, k, 0)+\overline{\Delta D}(i+1, k, 1)$

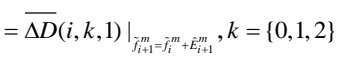

Wherein $\tilde{f}_{i+1}^{m}$ refers to the predicted value to which the $\mathrm{m}^{\text {th }}$ pixel of $\mathrm{i}+1^{\text {th }}$ frame corresponds and $\hat{E}_{i+1}^{m} \quad$ refers to the information on difference value to which the pixel corresponds. Plug formulas (8) and (9) into formula (7), and calculate the average distortion variation value of whole GOP when $\mathrm{i}^{\text {th }}$ frame gets lost respectively in DPA, DPB and DPC.

After average distortion measure caused by the loss from data partitioning is obtainded with the above method, it is OK to assign them different important factors. Determine two thresholds $D_{1}$ and $D_{2}$ : Set the importance factor $I F$ as High when the avarage distortion is greater than $D_{1}$ and set $I F$ as 
Medium when between $D_{1}$ and $D_{2}$; otherwise set $I F$ as Low, as shown in the formulas below:

$\left\{\begin{aligned} I F=\text { High }: & \overline{\Delta D}(i, k) \geq D_{1} \\ I F=\text { Medium }: & D_{2} \overline{\Delta D}(i, k) \leq D_{1} \\ I F=\text { Low }: & \overline{\Delta D}(i, k)<D_{2}\end{aligned}\right.$

In this way, the mapping mechanism is made as shown in Fig.2 in the light of calculation results from formula (10), which maps the video data of different importance to different queues $A C_{i}$.

Competition bewteen sites will not be repeated in this paper. Transmission for the different types of H.264 video data packets is simulated in this paper with the purpose of finding out the packet scheduling between sites in various queues. For simplicity, the static mapping is adopted as shown in Fig. 1 about mapping of the video packet. Wherein parameter set (i.e. NVB) includes the sequence parameter set (SPS) and the picture parameter set (PPS), and IDR is an independently coded frame. During the data partitioning, the IDR frame is not devided while the P-frame is divided into DPA, DPB and DPC. Video scheduling inside the sites is shown as Fig. 1 in which the label mode of each frame and partitioning is "Frame number Frame type_Partition type Partion number" For instance, 2P_A1 indicates the first A-partition of the second frame; 2P_A2 indicates the the second A-partition of the second frame. It is evident that the parameter set is data of the highest degree of importance and enters the highest-priority queue $A C_{3}$, so it is the first scheduled. IDR-partition and A-partition lies in the next highest priority level, then comes the the second frame - P-frame. In order of the priority, A1 is firstly didvided, then B1, followed by A2 and A3, which indicates the second priority level is first scheduled rather than the third priority level. The video packet will enter its own queue by the fixed priority just as the mapping mechanism shown in Table 1. Besides, relatively speaking, the partitioned data of IDR and A has a smaller share in total data than that of $\mathrm{B}$ and $\mathrm{C}$ partitions. Under this static mapping mechanism, paremeter set, IDR and $\mathrm{A}$ partitions are always divided in the first place, therefore, the high-priority queues is of low uitilization efficiency.

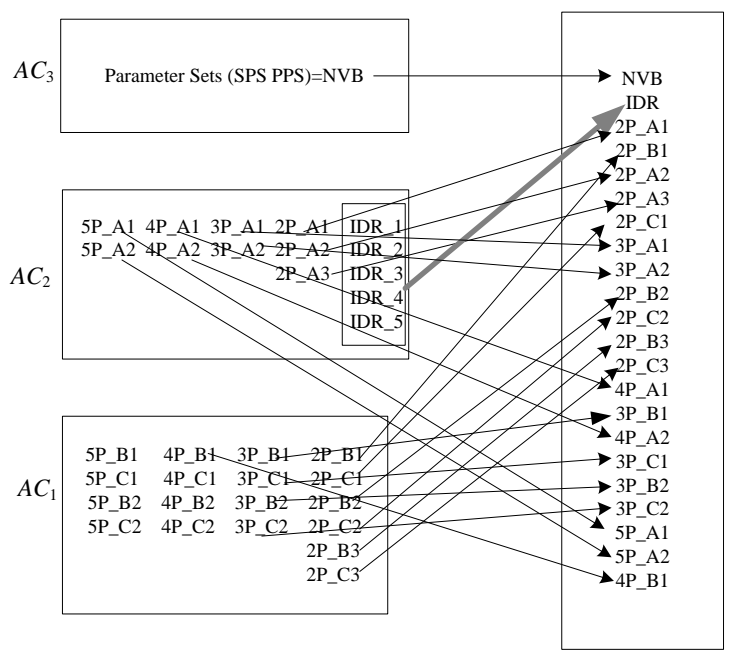

Fig. 1 Analysis diagram of 802.11e queue scheduling

\section{Simulation results and analysis}

Adopt JM16.0 software to encode and decode the video sequence in the simulation experiment, and select the typical sequence "foreman", CIF format, frame rate $30 \mathrm{fps}$, and the length of GOP 16. After encoding, generate data file of the video packet video_file in which the information on video packet is stored, such as ID number, size, transmission time and encoding type. The wireless network environment of 802.11e is simulated with NS2.29 including one wireless access point AP and 5 radio transceiver terminals sta0 4 in the simulation scene. The data flow is simulated with the wireless access point $\mathrm{AP}$ transmiting data packet respectively to sta0 sta4 through $A C_{0} \sim A C_{3}$ queues, sending video flow to sta0 and CBR background flow of $300 \mathrm{kps}$ rate to sta1 4. The basic parameter setting (queue setting) of 802.11e is shown in Table 3. 
Table 3 Basic parameter setting of 802.11e

\begin{tabular}{ccccc}
\hline queue & $\begin{array}{c}\text { priority } \\
\text { level (0 is } \\
\text { the highest } \\
\text { priority) }\end{array}$ & $\begin{array}{c}\text { AIFS } \\
\text { (us) }\end{array}$ & CWmin & CWmax \\
\hline$A C_{3}$ & 0 & 50 & 7 & 15 \\
$A C_{2}$ & 1 & 50 & 15 & 31 \\
$A C_{1}$ & 2 & 50 & 31 & 1023 \\
$A C_{0}$ & 3 & 70 & 31 & 1023 \\
\hline
\end{tabular}

Read content in the data file of video packet video_file into NS2 event scheduler and output the transmission results of video packet with the aid of NS2-simulated network environment. Statistically analyze the teansimission results to obtain the packet loss rate and packet delay of the video packet. From the perspectives of packet loss rate and video reconstruction quality, the three algorithms, i.e. EDCA default mapping algorithm, static mapping algorithm in document [2] and algorithm in this paper are compared as shown in Table 4 which shows the EDCA default mapping algorithm falis to prioritize different video data resulting in all the video packets being mapped to $A C_{2}$ queue and that is why the experiment results are all about the total average packet loss rate of the video packet. For the impotant IDR and DPA data, the packet loss rate is 0 with both the static mapping algorithm in document [2] and algorithm in this paper, which demonstates that these two aglorithms is highly protective of the important data. In terms of DPB and DPC data, when $Q P=27$, the packet loss rate of DPB data is $11.49 \%$ with aglorithm in the document [2] while $8.32 \%$ with aglogirthm in this paper, reducing 3.17\%; the packet loss rate of DPC data is $18.85 \%$ with aglorithm in the document [2] while $16.35 \%$ with aglogirthm in this paper, reducing $2.5 \%$. when $Q P=30$, the packet loss rate of DPB data is $11.63 \%$ with aglorithm in the document [2] while $9.27 \%$ with aglogirthm in this paper, reducing $2.36 \%$; the packet loss rate of DPC data is $19.04 \%$ with aglorithm in the document [2] while $17.61 \%$ with aglogirthm in this paper, reducing $1.43 \%$. Therefore, the packet loss rate cuts down for both the IDR and DPA data, even the less important DPB and DPC data with the algorithm put forward in this paper. Seen from the video reconstruction quality, the method in paper is of the highest PSNR value, $2 \mathrm{~dB}$ greater than that of the EDCA default mapping algorithm and $0.8 \sim 1.3 \mathrm{~dB}$ greater than that of the static mapping algorithm in document [2].

Table 4 Performance evaluation of different mapping approach

\begin{tabular}{|c|c|c|c|c|c|c|}
\hline $\begin{array}{l}\text { foreman } \\
\text { sequence }\end{array}$ & $\begin{array}{l}\text { Performance } \\
\text { parameter }\end{array}$ & $\begin{array}{c}\text { EDCA } \\
\text { algorithm }\end{array}$ & $\begin{array}{c}\text { static m } \\
\text { in }\end{array}$ & $\begin{array}{l}\text { algorithm } \\
\text { nt [2] }\end{array}$ & Algor & $\begin{array}{l}n \text { in this } \\
\text { er }\end{array}$ \\
\hline \multirow{6}{*}{$Q P=27$} & \multirow{3}{*}{ packet loss rate } & I & IDR & 0.0000 & IDR & 0.0000 \\
\hline & & / & DPA & 0.0000 & DPA & 0.0000 \\
\hline & & / & DPB & 0.1149 & DPB & 0.0832 \\
\hline & \multirow{3}{*}{$\begin{array}{l}\text { The total average } \\
\text { packet loss rate } \\
\text { Average PSNR }\end{array}$} & / & DPC & 0.1885 & DPC & 0.1635 \\
\hline & & 0.0693 & \multicolumn{2}{|c|}{0.0541} & \multicolumn{2}{|c|}{0.0273} \\
\hline & & 35.71 & \multicolumn{2}{|c|}{36.42} & \multicolumn{2}{|c|}{37.22} \\
\hline \multirow{6}{*}{$Q P=30$} & \multirow{3}{*}{ packet loss rate } & I & IDR & 0.0000 & IDR & 0.0000 \\
\hline & & / & DPA & 0.0000 & DPA & 0.0000 \\
\hline & & / & DPB & 0.1163 & DPB & 0.0927 \\
\hline & & / & DPC & 0.1904 & DPC & 0.1761 \\
\hline & $\begin{array}{l}\text { The total average } \\
\text { packet loss rate }\end{array}$ & 0.0736 & \multicolumn{2}{|c|}{0.0553} & \multicolumn{2}{|c|}{0.0311} \\
\hline & Average PSNR & 33.43 & \multicolumn{2}{|c|}{34.15} & \multicolumn{2}{|c|}{35.48} \\
\hline
\end{tabular}




\section{Conclusions}

The video optimization transmission algorithm is proposed comprehensively considering the unequal importance of video information and EDCA queue state. Above all, influence from the loss of $\mathrm{A}, \mathrm{B}$ and $\mathrm{C}$ partitioned data on the distortion are quantatively analyzed, from which the importance factors are obtanined. Then, in view of importance factors and queue length, the video packet is maaped to different EDCA queues to provide the video data packet with difference services. The simulation results indicate that the algorithm in this paper enhance the video reconstruction quality and improve the tranmission performance of video flow on WLAN when compared to default mapping algorithm of 802.11e EDCA and static mapping algorithm in document [2].

\section{Reference}

[1] MacKenzie R, Hands D, O' Farrell T. QoS of video delivery over 802.11e WLANs [C]. //Proc of the IEEE International Conference on Communications, Dresden, Germany, June 2009: 1-5.

[2] A. Ksentini, M. Naimi, A. Gueroui, Toward an Improvement of H.264 Video Transmission over IEEE 802.11e through a Cross-layer Architecture [J]. IEEE communication magazine, 2006, 44(1): 107-114.

[3] Xiaofeng Li, Tonghua Ren, Jin Xu, A Cross-layer Design for Transmission of Scalable H.264 Video over IEEE 802.11e Networks [C]. //Proc of International Conference on Consumer Electronics. 2010: 306-309.

[4]Y. Geng, J. He, H. Deng and K. Pahlavan, Modeling the Effect of Human Body on TOA Ranging for Indoor Human Tracking with Wrist Mounted Sensor, 16th International Symposium on Wireless Personal Multimedia Communications (WPMC), Atlantic City, NJ, Jun. 2013.

[5]Y. Geng, J. He, K. Pahlavan, Modeling the Effect of Human Body on TOA Based Indoor Human Tracking[J], International Journal of Wireless Information Networks 20(4), 306-317 